Supporting Information for

\title{
Density of obstacles affects diffusion in adsorbed polymer layers
}

Cristian Rodríguez-Tinoco, ${ }^{1}$ David Nieto Simavilla ${ }^{1}$, Rodney D. Priestley, ${ }^{2}$ Michael Wübbenhorst ${ }^{3}$ and Simone Napolitano ${ }^{1, *}$

${ }^{1}$ Laboratory of Polymer and Soft Matter Dynamics, Experimental Soft Matter and Thermal Physics (EST), Faculté des Sciences, Université libre de Bruxelles (ULB), Boulevard du Triomphe, Bruxelles 1050, Belgium

${ }^{2}$ Department of Chemical and Biological Engineering, Princeton University, Princeton, New Jersey 08544, United States

${ }^{3}$ Department of Physics and Astronomy, Soft Matter and Biophysics Section, KU Leuven, Celestijnenlaan 200D, B-3001 Leuven, Belgium

*email: snapolit@ulb.ac.be

A. Synthesis of the polar probes I-PS

B. Annealing protocol used for the preparation of the adsorbed layers 


\section{Synthesis of the polar probes, I-PS}

4-Aminobenzonitrille (Aldrich), 2-(methylphenylamino)ethanol (Aldrich), methacrylic anhydride (Aldrich), styrene (Sigma-Aldrich), and 2,2'-azoisobutyronitrile (AIBN) (Fluka) were used as received. The synthesis of the chromophore 4-[(E)-[4-(2hydroxyethyl(methyl)amino)phenyl]azo]benzonitrile (CN) was carried out according to literature procedures [S1]. The photochromic methacrylate 2-[4-[(E)-(4-cyanophenyl)azo]$\mathrm{N}$-methylanilino]ethyl 2-methacrylate (MCN) was obtained starting from azo dye (CN) and methacrylic anhydride and following the reaction elsewhere described [S2]. The labeled polystyrene, poly(2-[4-[(E)-(4-cyanophenyl)azo]- $N$-methylanilino]ethyl 2-methacrylate-costyrene), was synthesized in bulk radical polymerization in the presence of azobis(isobutyronitrile) (AIBN), used as radical initiator. The reaction was performed in an ampule, purged with nitrogen, where the styrene monomer was first mixed with the methacrylic monomer $(\mathrm{MCN})(3 \% \mathrm{~mol})$ and then with the AIBN initiator $(0,15 \% \mathrm{w} / \mathrm{w})$. The mixture was kept at $90^{\circ} \mathrm{C}$ in a nitrogen atmosphere for $72 \mathrm{~h}$. After completion of the reaction, the copolymer was dissolved in benzene and purified by precipitation in methanol. Gel permeation chromatography (GPC) measurements revealed that the functionalized polystyrene (I-PS) had a $M_{\mathrm{w}}=20 \mathrm{~kg} / \mathrm{mol}$ and PDI $=2$. 


\section{Annealing protocol used for the preparation of the adsorbed layers}

Irreversibly adsorbed layers, used as PS matrices, were prepared using a standard procedure [S3]. Thick films ( > 7Rg, typically $300 \mathrm{~nm}$ ) of neat monodisperse (PDI $\leq 1.06)$ PS samples of different Mw spincoated on aluminum were annealed for different times at $\mathrm{T}_{\mathrm{g}}$ matrix $+60 \mathrm{~K}$. Subsequent washing of the samples in the same good solvent (chloroform) used for spincoating allowed removal of non-adsorbed chains. Irreversibly adsorbed layers with thicknesses, $h_{\text {ads, }}$ ranging from 3.8 to $8.5 \mathrm{~nm}$ were prepared by varying the annealing time $t_{\text {ANN }}$ and $M_{w}$. The variation of the thickness of the adsorbed layer with annealing time is given in Figure S1.

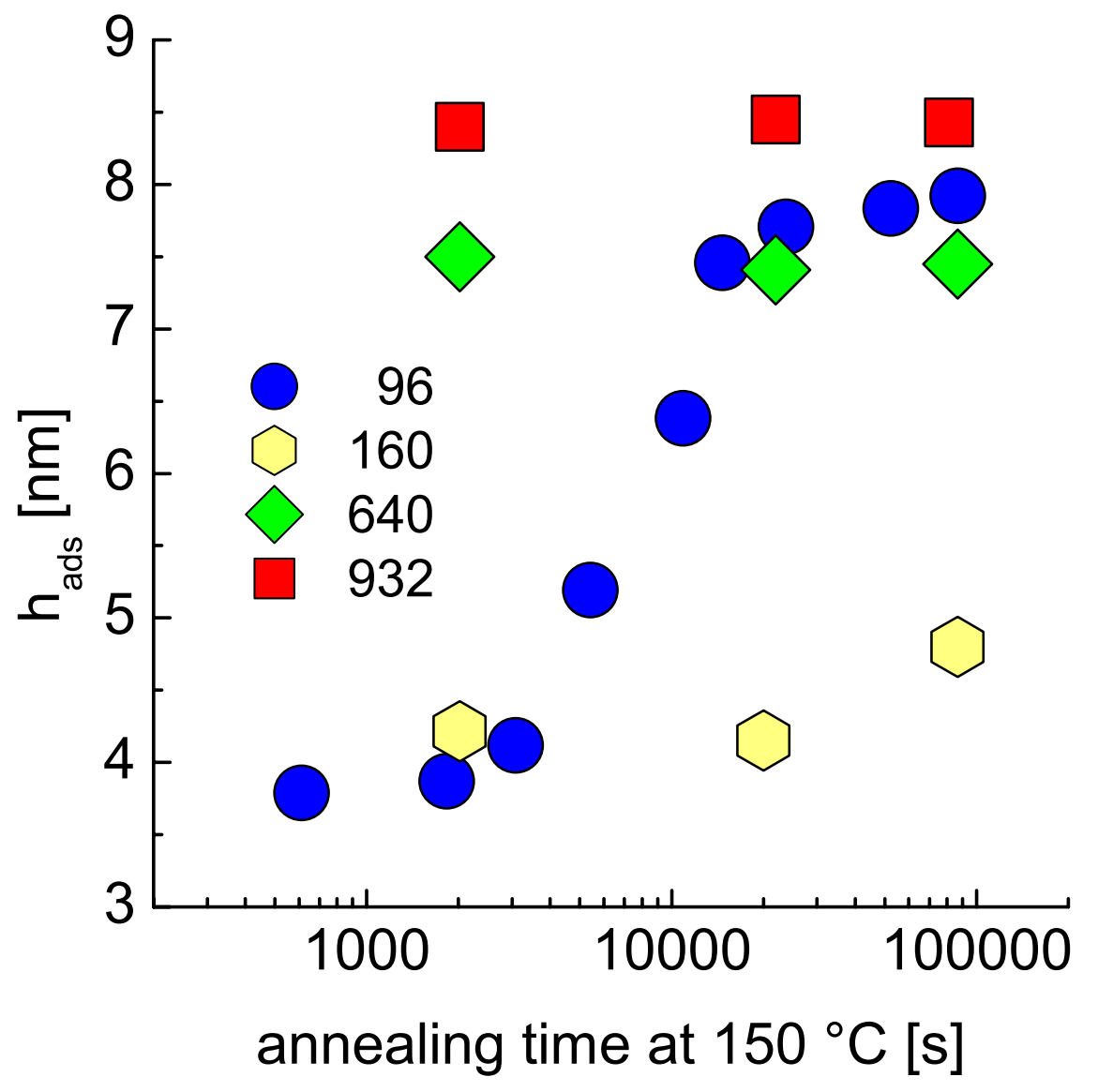

Figure S1. Kinetics of adsorption for melts of PS on Al. The legend contains the molecular weight in $\mathrm{kg} / \mathrm{mol}$.

[S1] Serwadczak, M.; Kucharski, S. Photochromic Gratings in Sol-Gel Hybrid Materials Containing Cyanoazobenzene ChromophoresJ. Sol-Gel Sci. Technol. 2006, 37, 57-62.

[S2] Zielinska, S.; Ortyl, E.; Barille, R.; Kucharski, Preparation and characteristics of new chiral photochromic copolymers, S. Opt. Mater. 2009, 32, 198-206.

[S3] Simavilla, D. N.; Panagopoulou, A.; Napolitano, S., Characterization of Adsorbed Polymer Layers: Preparation, Determination of the Adsorbed Amount and Investigation of the Kinetics of Irreversible Adsorption. Macromolecular Chemistry and Physics 2017, 219, 201700303. 